\title{
Journal-to-journal shortcuts
}

\author{
At Nature Publishing Group we offer a transfer system that allows authors to move papers between \\ our journals at the click of a button if their first-choice journal declined. We encourage authors to use \\ that service.
}

Peer review helps to improve papers, and to select those that are robust. But it can also delay the publication of quality research, and be a drain on the time of both authors and referees. This is especially true when publication of a paper is ultimately declined because it does not meet the editorial standards for novelty and importance of the chosen journal. For Nature, the Nature research journals and Nature Communications, authors can take the option to transfer a submission between journals and salvage the time that authors and referees spent on a paper. For example, if a manuscript is declined by Nature after review, it can be transferred, along with referees' reports and identities, to Nature Geoscience (or likewise from Nature Geoscience to Nature Communications).

To us, manuscripts that come from Nature with referees' reports are very welcome. We have much more information on the work compared with a fresh, new submission. So we can make a decision on the likely prospect of a future revised version, usually within a week or so, without first having to consult with referees. If we, too, decline, the authors have not lost much time. If we are interested, we can simply take the peer review process forward from where Nature left off. For the authors, that means that they can work towards improving the manuscript with the same set of referees, and could save a round or two of review.

For this process to work as efficiently as possible, we encourage authors to transfer the latest version of the paper that was seen by referees (but declined) - ideally together with a brief outline of ways to address the remaining criticisms. Because referee comments and identities are transferred along with the paper, this information allows us or, if the transfer is from Nature Geoscience, the editors of the receiving journal - to immediately evaluate the manuscript and review comments according to our own editorial criteria with the benefit of a full review by referees.

The outcome of this initial assessment is usually clear advice from us, specifying the minimum set of conclusions in the paper that need to be compelling in order for the paper to suit the journal. In general, we do

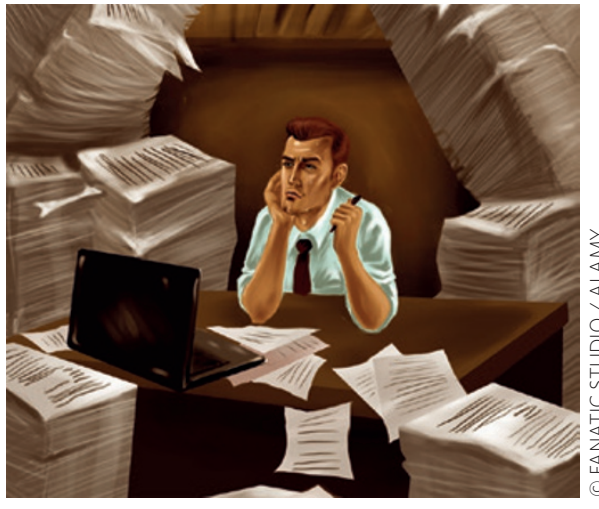

Regardless of how a manuscript comes to us - as a transfer from another journal or as a new submission - we read the paper and any relevant correspondence and make our own editorial assessment. Transfer status is neither an advantage nor a disadvantage: in 2013 the accept rate among all submissions was very similar to the accept rate among manuscripts that had been transferred from Nature. All technical issues in a paper need to be addressed, the difference between journals lies solely in the specific editorial requirements. Broadly speaking, our criteria in terms of novelty and importance are slightly more relaxed than those at Nature, and stricter than

Scientists need relief from the pressure to review. at Nature Communications.

The decision to transfer a manuscript before or after review - lies exclusively with the authors. As editors of Nature Geoscience, we may make a recommendation for another journal that we feel might be more suitable, as part of our decision letter. This suggestion is non-committal in both directions: authors are free to follow the suggestion or not, and we do not guarantee that the editors of the receiving journal will pursue the paper. Nevertheless, we have a good idea of the editorial criteria of our sister journals: for example, a large majority of the papers that were transferred from Nature Geoscience to Nature Communications in the past half year with a recommendation from us were deemed suitable for the journal, in principle, and sent out for peer review.

Usually, when publication of a paper is declined at the journal of their choice, the authors then have to start again from scratch elsewhere. Manuscripts must be reformatted, and new files uploaded. New referees have to read the paper afresh, and then often raise new concerns that may even contradict some of the earlier comments the authors received. This is, of course, not surprising. Referees are human, too, and have their own perspectives and their own specific sets of interests and expertise.

We hope that our transfer system helps to streamline the authors' experience and at the same time alleviate the increasing pressure on scientists to review papers. We want to help authors to publish their work as swiftly as possible. In this, we do not compromise on quality, but we want to offer a shortcut around any unnecessary duplication of effort. 